\title{
Changes of Musculoskeletal Deformity in Severely Disabled Children Using the Custom Molded Fitting Chair
}

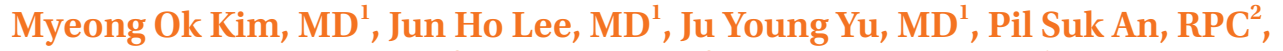 \\ Do Hang Hur ${ }^{2}$, Eun Seo Park ${ }^{2}$, Jae Hong Kim, MD ${ }^{1}$ \\ ${ }^{1}$ Department of Physical \& Rehabilitation Medicine, Inha University School of Medicine, Incheon; \\ ${ }^{2}$ Technical Aid Center, Notre Dame Welfare Center, Incheon, Korea
}

Objective To know the effectiveness of a custom molded fitting chair between pre- and post-chair status through comparison of musculoskeletal indices in severely disabled children.

Methods We researched 34 severely disabled patients who had used a custom molded fitting chair continuously for more than a year. There were 27 cerebral palsy patients and 7 patients with other kinds of diseases that affect the brain such as chromosomal disease or metabolic disease. By radiographic studies, Cobb's angle, the femoral neck-shaft angle of the femur, and Reimers migration percentage were measured. The indices are analyzed before and after application.

Results The average period of application was 24 months. There was a significant reduction in the angles of femur neck-shaft, 163.4 degree before and 158.2 degree after the use of the chair $(\mathrm{p}<0.05)$, and 23 of 34 had demonstrated a reduced angle. Cobb's angle and Reimers migration percentage increased but the difference of pre- and postchair status was not statistically significant. Seventeen of 33 children showed reduced Cobb's angle. Also, 19 of 37 showed a reduced degree of dislocation of the hip joints.

Conclusion In spite of the use of a custom molded fitting chair, a significant improvement did not emerge for musculoskeletal deformity indices in severely disabled children. However, there was no significant aggravation of Cobb's angle or Reimers migration percentage in developing children. Therefore, it is thought be helpful to prevent rapid aggravation of musculoskeletal deformities.

Keywords Orthotic devices, Musculoskeletal system, Disabilities, Disabled children

Received March 27, 2012; Accepted August 21, 2012

Corresponding author: Jae Hong Kim

Department of Physical \& Rehabilitation Medicine, Inha University School of Medicine, 27 Inhang-ro, Jung-gu, Incheon 400-711, Korea

Tel: +82-32-890-2480, Fax: +82-32-890-2486, E-mail: recolo2009@gmail. com

(c) This is an open-access article distributed under the terms of the Creative Commons Attribution Non-Commercial License (http://creativecommons. org/licenses/by-nc/3.0) which permits unrestricted noncommercial use, distribution, and reproduction in any medium, provided the original work is properly cited.

Copyright $\odot 2013$ by Korean Academy of Rehabilitation Medicine

\section{INTRODUCTION}

Severely disabled children suffer from a lot of musculoskeletal deformities. The diseases that cause musculoskeletal deformities are heterogeneous. It includes not only cerebral palsy (CP), but also myopathy, metabolic disease, motor neuron disease, and other various congenital diseases.

The prevalence of scoliosis in patients with spastic CP 
ranges from $15 \%$ to $61 \%$. Hip subluxation and dislocation are the second most common deformities in patients with spastic $\mathrm{CP}$, with a reported prevalence of up to $28 \%$. Associated abnormalities include pelvic obliquity (68\%), hip contractures $(79 \%)$, and femoral head subluxation (59\%). The most commonly affected muscles are the paraspinal muscles, hip flexors, hip adductors, hamstrings, gastrocnemius, and soleus [1].

In severely disabled children, scoliosis is one of the most common musculoskeletal deformities and is considerably worse compared to mildly disabled children. Curve progression of $0.8^{\circ}$ per year when the largest curve was less than or equal to $50^{\circ}$ at skeletal maturity and curve progression of $1.4^{\circ}$ per year when the largest curve was greater than $50^{\circ}$ at skeletal maturity [2].

As scoliosis shows the gradual increase of the deformed angle, it may induce pain, emotional disorder, pressure sores, cardiopulmonary dysfunction, and other secondary problems [3]. In contrast with idiopathic scoliosis, it has a tendency to progress from a flexible curvature to a permanent deformity because the progression continues even after the end of growth. Particularly, in cases with pelvic obliquity, the prognosis may become worse [4,5]. A large number of severely disabled children use the custom molded fitting chair that is manufactured for their specific body shape, because the premanufactured offthe-shelf component or fabric-based sling seat are not inclined for that purpose. Although disabled children surely need the orthotics for transfer, study, or nursing, they cannot use the ready-made or fabricated orthotics for their deformities. Their deformities are too severe to use the premanufactured or fabric-based type seat. Also, the chair is helpful to reduce the effort of the caregiver allowing the patient to sit comfortably for hours.

The chairs were manufactured in the Technical Aid Center of the Notre Dame Welfare Center. Before manufacturing the chair, the patients visited our hospital and were examined in regards to their disease, lifestyle, and musculoskeletal disabilities. The orthotics that can be manufactured are buggy, postural wheelchair (PW), wheelchair, car seat, chair for study, and so on. After determining which type of orthotics would be made, the inner is manufactured by the molding system. After laying the patient on the simulator, a suitable shape is created. Then, the simulator transmits the plane image of the shape to the computer. Once transmitted, the computer reconstructs the three dimensional image from the plane image, and the technician makes the inner according to the blueprint (Fig. 1). The arm board or headrest is added according to the kinds of orthotics and severity of the disability. The suspending belt for the pelvis or trunk is also attached. After manufacturing the inner, it is installed to the frame of the orthotics such as a wheelchair (Fig. 2).

When manufacturing the chairs, for their comfort, the physiatrist should consider the musculoskeletal deformity of the patients. The rapid growth in adolescents is likely to cause musculoskeletal deformity and several other complications in adulthood [6]. The most common problem is deformities of the spine and hip joint in patients with CP [1]. Scoliosis is defined as a deformity where the spine is deviated laterally or rotated. Cobb's angle is the most popular index to evaluate the severity of scoliosis [7]. Hip dislocation is also common in children with CP. Reimers migration percentage is the index to assess the severity of hip dislocation or subluxation [8]. Coxa valga has appeared in most hip joints of CP, and is evaluated with the femoral neck-shaft angle of the femur. The angle varies with age, stature, and width of the pelvis, being less in adults. When the angle is larger than $135^{\circ}$, condition is known as coxa valga [9].

We followed up the indices of musculoskeletal deformity in severely disabled children to investigate the effectiveness of custom molded fitting through comparison of musculoskeletal indices between pre- and post-chair status, and to determine the correlation between three indices of musculoskeletal deformity.

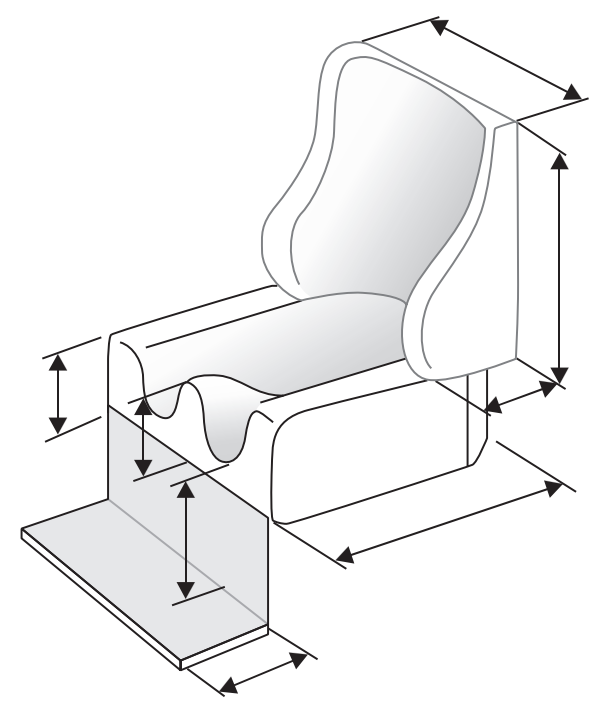

Fig. 1. The blueprint making an inner for the chair. 

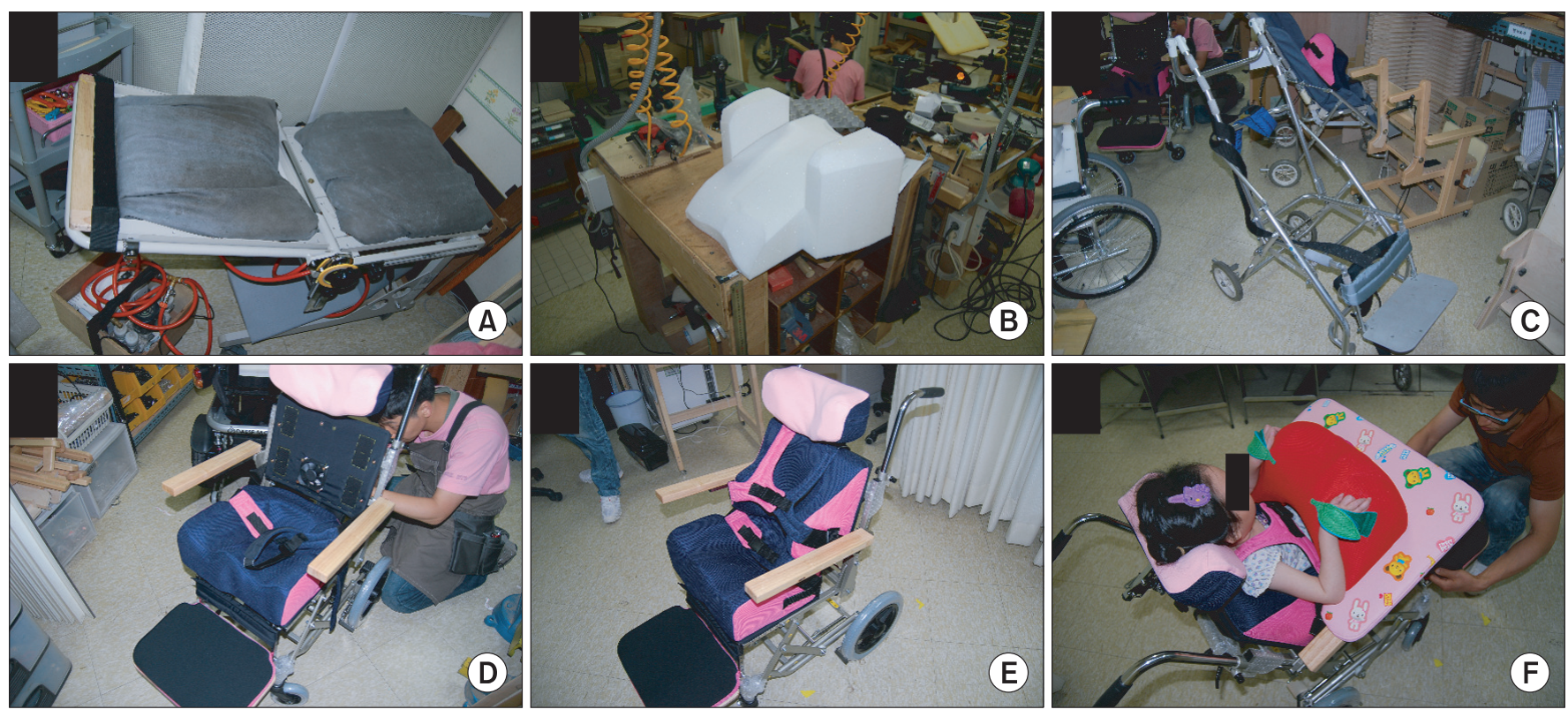

Fig. 2. Manufacturing process of the custom molded fitting chair. Simulator scanned the body shape of the patient (A). The inner was reconstructed from the image scanned by the simulator (B). A frame of a manual wheelchair was selected to apply the inner (C). The seat portion of the inner was installed to the frame. A fan is attached under the back for ventilation (D). The back portion was installed (E). Applying the patient to the chair, a few adjustments were needed (F).

\section{MATERIALS AND METHODS}

\section{Subject}

Thirty-four severely disabled patients (19 males and 15 females) using the custom molded fitting chair continuously participated in this study for several years. The mean age of the subjects was 8.6 years (range, 4 to 20 years). There were $27 \mathrm{CP}$ patients and 7 patients with other kinds of diseases that affect the brain such as DandyWalker syndrome, Arnold-Chiari malformation, congenital metabolic disease, and so on (Table 1). All the patients were severely disabled and the Gross Motor Function Classification System (GMFCS) of the children was all 5. Patients using the custom molded fitting chair for less than a year were excluded, and the average period of application was 24 months. The average fitting time of the chair was 3.7 hours (range, 0.5 to 8 hours) a day.

\section{Method}

We performed a radiographic examination of the spine and pelvis before and after the use of the chair. Cobb's angle, Reimers migration percentage, and the femoral neck-shaft angle of the femur were calculated by using plain radiographic images. The radiographic examination was done before and after the use of the chair. The
Table 1. Diseases of the patients and type of cerebral palsy (CP)

\begin{tabular}{lr}
\hline \multicolumn{1}{c}{ Disease (type of CP) } & No. \\
\hline CP spastic bilateral & \\
\hline (Quadriplegia) & 20 \\
\hline (Diplegia) & 4 \\
CP spastic unilateral & 1 \\
CP dyskinetic (dystonic) & 1 \\
CP nonclassifiable & 1 \\
\hline Microcephaly with hypotonia & 2 \\
\hline Dandy-Walker syndrome & 1 \\
\hline Sturge-Weber syndrome & 1 \\
\hline Chromosomal anomaly with hypotonia & 1 \\
\hline Arnold-Chiari malformation type I & 1 \\
Lesch-Nyhan syndrome & 1 \\
Total & 34 \\
\hline
\end{tabular}

average interval period between the initial and follow-up radiologic workup was 24 months. To prove the effectiveness of the chair, we analyzed the findings of improvement and maintenance for these indices. All patients were examined on the supine position because of their severe disability. To clarify the influence of the growth spurt period, we divided the patients into two groups: 
growth spurt group (10-15 years old) and non-growth spurt group. In addition, we analyzed the effectiveness of the chair according to fitting time. In addition, these 34 patients were divided into two groups: short time fitting group (less than 3.7 hours) and long time fitting group (more than 3.7 hours). The average fitting time was 3.7 hours.

Cobb's angle was measured to choose the most tilted vertebrae above and below the apex of the curve. It is the angle between intersecting lines drawn perpendicular to the top of the top vertebrae and the bottom of the bottom vertebrae (Fig. 3). Reimers migration percentage was measured to determine the lateral displacement of the femoral head from its normal position in the acetabulum. It is the percentage of the femoral head lying lateral to Perkins' line (P), which is drawn perpendicular to Hilgenreiner's line (H) (Fig. 4). The femoral neck-shaft angle was measured to assess of coxa valga of the hip joint. The angle is formed by 2 lines. One is drawn through the femoral shaft midway; the other is drawn through the center
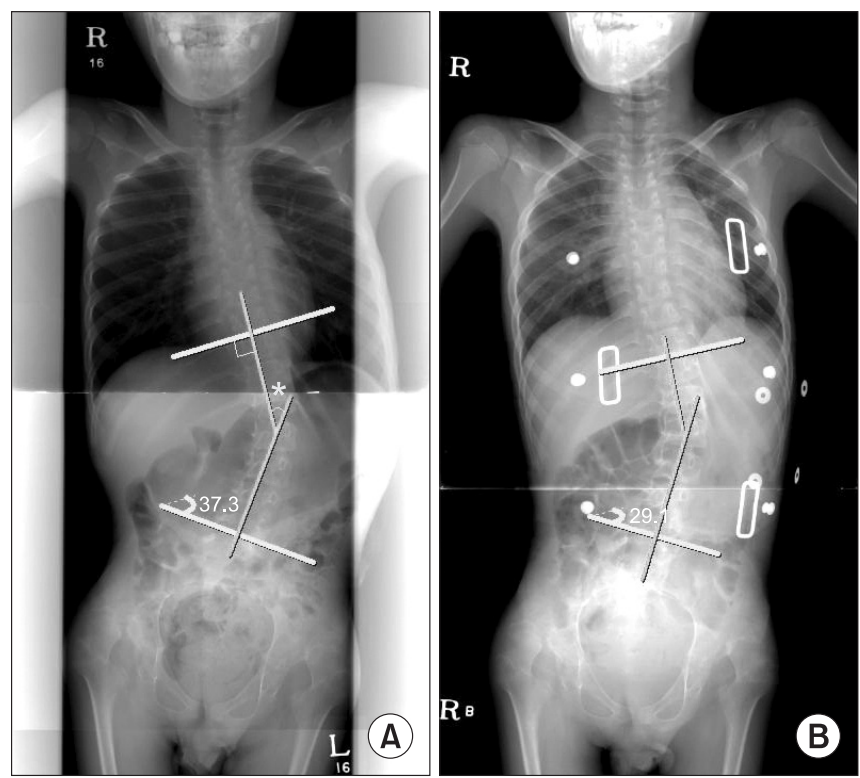

Fig. 3. Cobb's angle $\left({ }^{*}\right)$ measurement in scoliosis. (A) Before application of the chair. (B) After application of the chair.
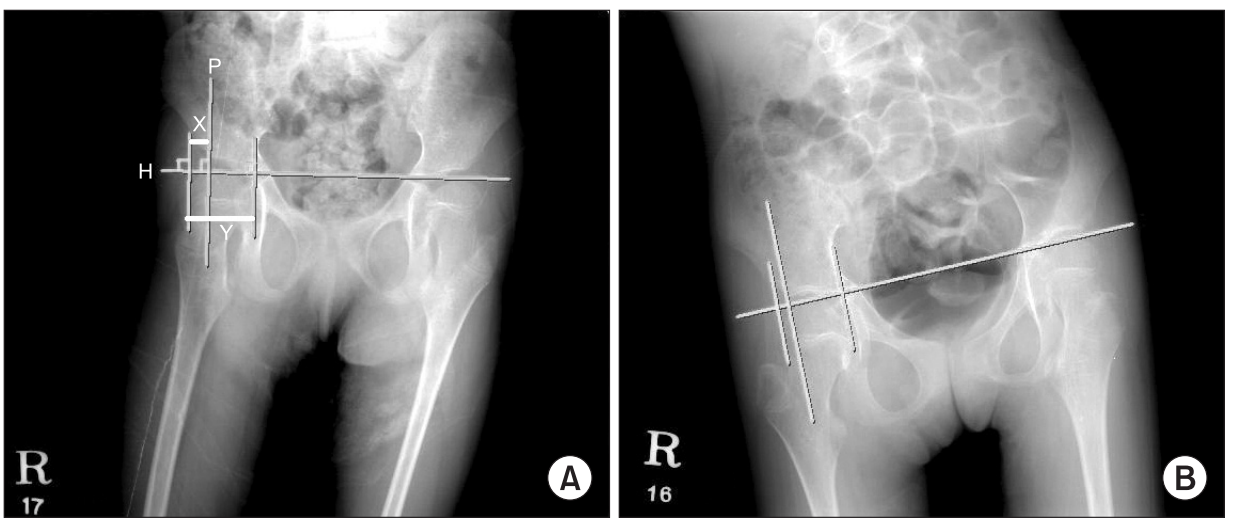

Fig. 4. Reimers migration percentage in hip subluxation. Perkins' line $(\mathrm{P})$, which is drawn perpendicular to Hilgenreiner's line $(H)$. Reimers migration percentage is the percentage of the femoral head lying lateral to Perkins' line (P). Reimers migration percentage $=\mathrm{X} /$ $Y \times 100$. (A) Before application of the chair. (B) After application of the chair.
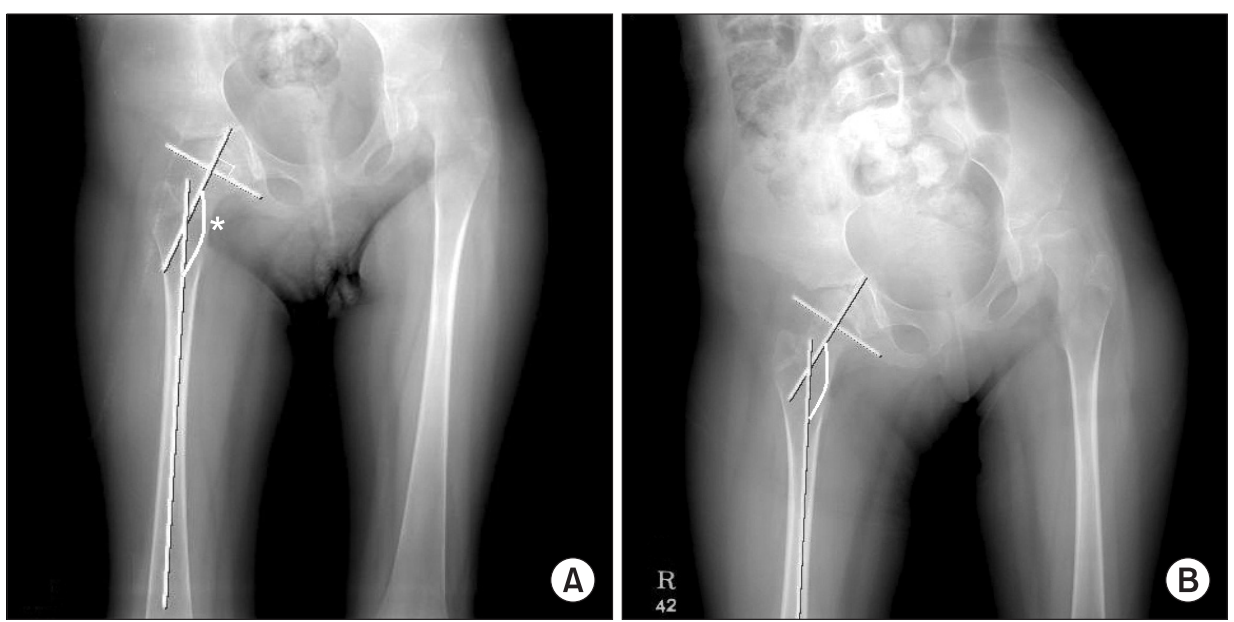

Fig. 5. Femoral neck-shaft $(*)$ angle in coxa valga. (A) Before application of the chair. (B) After application of the chair. 
of the proximal femoral epiphysis and perpendicular to the physis. The angle becomes narrower with age (Fig. 5).

\section{Statistical analysis}

SPSS ver. 12.0 (SPSS Inc., Chicago, IL, USA) was used for statistical analysis. To evaluate the effect of the custom molded fitting chair before and after application, all musculoskeletal indices were analyzed by paired t-test. We analyzed the paired t-test between pre- and post-chair fitting during the growth spurt period and non-growth spurt period. The results were found to be statistically significant at $\mathrm{p}<0.05$.

\section{RESULTS}

\section{Changes of musculoskeletal indices}

The Cobb's angle measured before application of the chair was $12.3 \pm 14.9^{\circ}$. After application of the chairs, this increased to $13.9 \pm 18.9^{\circ}$, but was not significantly different. Reimers migration percentage measured before application of the chairs was $32.2 \pm 21.4 \%$. After application

Table 2. Changes of musculoskeletal indices

\begin{tabular}{lcc}
\hline & Pre-chair & Post-chair \\
\hline Femoral neck-shaft angle $\left(^{\circ}\right)$ & $163.4 \pm 9.9$ & $158.2 \pm 12.3^{*}$ \\
Cobb's angle $\left(^{\circ}\right)$ & $12.3 \pm 14.9$ & $13.9 \pm 18.9$ \\
\hline $\begin{array}{l}\text { Reimers migration percentage } \\
\quad \%)\end{array}$ & $32.2 \pm 21.4$ & $35.1 \pm 25.9$ \\
\hline
\end{tabular}

Values are presented as mean \pm standard deviation. ${ }^{*} \mathrm{p}<0.05$. of the chairs, these are increased to $35.1 \pm 25.9 \%$, but not significant. The femoral neck-shaft angle was significantly reduced. Before application of the chair, the angle was $163.4 \pm 9.9^{\circ}$. However, after application of the chairs it was reduced to $158.2 \pm 12.3^{\circ}$ (Table 2 ).

Numbers of improved patients following application of the custom molded fitting chair

The numbers of improved patients after application of the chair were counted. Of the 34 patients, improvement of the femoral neck-shaft angle was observed in 23 patients. In addition, improvement or no aggravation of Cobb's angle and Reimers migration percentage were observed in 17 and 22 patients, respectively (Table 3). Aggravated patients were 16 and 15 patients in each index. The numbers of not aggravated patients were slightly more than aggravated patient.

Correlation analysis between musculoskeletal indices after application of the chair

In the correlation coefficient analysis between muscu-

Table 3. Post application status of fitting custom molded chair in 36 patients

\begin{tabular}{lccc}
\hline & Improved & No change Aggravated \\
\hline $\begin{array}{l}\text { Femoral neck-shaft } \\
\text { angle }\left(^{\circ}\right)\end{array}$ & 23 & 0 & 11 \\
$\begin{array}{l}\text { Cobb's angle }\left(^{\circ}\right) \\
\begin{array}{c}\text { Reimers migration } \\
\text { percentage (\%) }\end{array}\end{array}$ & 17 & 0 & 16 \\
\hline
\end{tabular}

Table 4. Correlation between musculoskeletal indices

\begin{tabular}{llcccccc}
\hline & & Pre-FSA & Pre-Cobb & Pre-RMP & Post-FSA & Post-Cobb & Post- RMP \\
\hline Pre-FSA & $\mathrm{r}$ & 1.000 & 0.118 & 0.215 & $0.537^{\mathrm{b})}$ & 0.156 & $0.431^{\mathrm{a})}$ \\
& $\mathrm{p}$-value & - & 0.529 & 0.222 & 0.001 & 0.402 & 0.011 \\
Pre-Cobb & $\mathrm{r}$ & 0.118 & 1.000 & $-0.381^{\mathrm{a})}$ & -0.162 & $0.878^{\mathrm{b})}$ & $-0.365^{\mathrm{a})}$ \\
& $\mathrm{p}$-value & 0.529 & - & 0.034 & 0.383 & 0.000 & 0.044 \\
Pre-RMP & $\mathrm{r}$ & 0.215 & $-0.381^{\mathrm{a})}$ & 1.000 & 0.243 & -0.349 & $0.684^{\mathrm{b})}$ \\
& $\mathrm{p}$-value & 0.222 & 0.034 & - & 0.167 & 0.054 & 0.000 \\
\hline Post-FSA & $\mathrm{r}$ & $0.537^{\mathrm{b})}$ & -0.162 & 0.243 & 1.000 & -0.206 & $0.518^{\mathrm{b})}$ \\
& $\mathrm{p}$-value & 0.001 & 0.383 & 0.167 & - & 0.266 & 0.002 \\
Post-Cobb & $\mathrm{r}$ & 0.156 & $0.878^{\mathrm{b})}$ & -0.349 & -0.206 & 1.000 & $-0.370^{\mathrm{a})}$ \\
& $\mathrm{p}$-value & 0.402 & 0.000 & 0.054 & 0.266 & - & 0.040 \\
\hline Post-RMP & $\mathrm{r}$ & $0.431^{\mathrm{a})}$ & $-0.365^{\mathrm{a})}$ & $0.684^{\mathrm{b})}$ & $0.518^{\mathrm{b})}$ & $-0.370^{\mathrm{a})}$ & 1.000 \\
& $\mathrm{p}$-value & 0.011 & 0.044 & 0.000 & 0.002 & 0.040 & - \\
\hline
\end{tabular}

FSA, femoral neck-shaft angle; Cobb, Cobb's angle; RMP, Reimers migration percentage.

${ }^{\text {a) }}$ Correlation is significant at the 0.05 level (2-tailed). ${ }^{\text {b) }}$ Correlation is significant at the 0.01 level (2-tailed). 
Table 5. Changes of musculoskeletal indices between growth spurt and non-spurt periods

\begin{tabular}{|c|c|c|c|c|}
\hline & \multicolumn{2}{|c|}{ Growth spurt period } & \multicolumn{2}{|c|}{ Non-spurt period } \\
\hline & Pre-chair & Post-chair & Pre-chair & Post-chair \\
\hline Femoral neck-shaft angle $\left(^{\circ}\right)$ & $162.7 \pm 10.2$ & $151.7 \pm 8.8^{*}$ & $163.7 \pm 9.9$ & $160.9 \pm 12.7$ \\
\hline Cobb's angle $\left(^{\circ}\right)$ & $17.8 \pm 14.2$ & $22.5 \pm 22.5$ & $10.0 \pm 14.9$ & $10.4 \pm 16.6$ \\
\hline Reimers migration percentage (\%) & $32.0 \pm 20.1$ & $26.5 \pm 18.0$ & $32.2 \pm 22.3$ & $38.7 \pm 28.1$ \\
\hline
\end{tabular}

Values are presented as mean \pm standard deviation.

${ }^{*} \mathrm{p}<0.05$.

Table 6. Effectiveness of the chair according to fitting time

\begin{tabular}{lcccccc}
\hline & \multicolumn{2}{c}{ Short time fitting } & & \multicolumn{2}{c}{ Long time fitting } \\
\cline { 2 - 3 } \cline { 5 - 6 } & Pre-chair & Post-chair & & Pre-chair & Post-chair \\
\hline Femoral neck-shaft angle $\left(^{\circ}\right)$ & $165.3 \pm 9.8$ & $162.5 \pm 11.8$ & & $162.5 \pm 11.2$ & $155.9 \pm 13.3$ \\
Cobb's angle $\left(^{\circ}\right)$ & $11.5 \pm 8.9$ & $14.6 \pm 17.3$ & & $12.2 \pm 17.3$ & $12.6 \pm 19.9$ \\
Reimers migration percentage $(\%)$ & $40.6 \pm 29.1$ & $46.7 \pm 30.1$ & & $27.1 \pm 10.1$ & $26.6 \pm 19.0$ \\
\hline
\end{tabular}

Values are presented as mean \pm standard deviation.

loskeletal indices after application of the chair, Reimers migration percentage was significantly correlated to the femoral neck-shaft angle $(\mathrm{r}=0.518, \mathrm{p}=0.002)$ (Table 4). Other correlations between the indices were not significant.

Changes of musculoskeletal indices between growth spurt and non-growth spurt periods

In the growth spurt group, the Cobb's angles before and after application of the chair were $17.8 \pm 14.2^{\circ}$ and $22.5 \pm 22.5^{\circ}$. Reimers migration percentage measured before and after application of the chair was $32.0 \pm 20.1 \%$ and $26.5 \pm 18.0 \%$. The femoral neck-shaft angle before and after application of the chair was $162.7 \pm 10.2^{\circ}$ and $151.7 \pm 8.8^{\circ}$. Thus, in the growth spurt group, there were statistically significant improvements in the femoral neck-shaft angle between pre- and post-application of the chair $(\mathrm{p}<0.05)$ (Table 5).

In the non-growth spurt periods, the Cobb's angles before and after application of the chair were $10.0 \pm 14.9^{\circ}$ and $10.4 \pm 16.6^{\circ}$. Reimers migration percentage measured before and after application of the chair was $32.2 \pm 22.3 \%$ and $38.7 \pm 28.1 \%$. The femoral neck-shaft angle before and after application of the chair was $163.7 \pm 9.9^{\circ}$ and $160.9 \pm 12.7^{\circ}$. In the non-growth spurt periods, there were no significant differences in all indices between pre- and post-application of the chair (Table 5).

\section{Effectiveness of the chair according to fitting time}

Cobb's angles were aggravated $-3.1^{\circ}$ and $-0.4^{\circ}$ in the long sitting group and short sitting group, respectively. Reimers migration percentage was aggravated $-6.1 \%$ in the short sitting group, but was improved $0.5^{\circ}$ in the long sitting group. The femoral neck-shaft angle was improved $2.8^{\circ}$ and $6.6^{\circ}$ in the short sitting group and the long sitting group, respectively (Table 6). However, there was no significant difference in all indices between the short and long sitting group.

\section{DISCUSSION}

A custom molded fitting chair is equipment used to aid in the retardation of aggravation of musculoskeletal deformities or unstable body posture caused by brain, spinal, and neuromuscular diseases. Another purpose of the custom molded fitting chair is to provide comfort to patients and lessen the burden of the caregiver. In practical terms, most caregivers get the chair for the comfort and improvement of activities of daily living for their children.

A previous study investigated the effect of the body shape molded inner system on the attenuation of spinal curvature and pelvic obliquity in cerebral palsy with scoliosis [10]. However, there is a limitation in that it only compares the spinal curvature after application of chair immediately. Therefore, a follow-up study of more than 1 year would prove to be more meaningful to know its 
clinical usefulness.

Not only spinal scoliosis, but hip dislocation is one of the important deformities in patients with CP. Hip dislocation may include seating difficulties, pain, gait deterioration, and problems with perineal hygiene [11]. Miller and Bagg [4] found that the hips of children with CP who were younger than 8 years and had a migration percentage of less than $30 \%$ were less likely to progress to dislocation than were the hips of children with a migration percentage of more than $30 \%$. If the migration percentage was greater than $60 \%$, complete dislocation of the hips without surgical intervention was reported [12]. The femoral neck-shaft angle is greater in children with CP than in typically developing children and is more pronounced in children with CP who are at risk for eventual subluxation [13]. It could be confirmed by positive correlation between Reimers migration ratio and femoral neck-shaft angle in this study because Reimers migration ratio indicates the degree of hip dislocation.

Although the femoral neck-shaft angle was improved after application of the chair, spinal scoliosis and hip dislocation was slightly aggravated. However, considering rapid aggravation of deformities with the growth spurt in adolescents, it should be reconsidered if the chair did not affect scoliosis or hip dislocation significantly. The rapid growth in adolescents is likely to cause musculoskeletal deformity, and complications may be accompanied in adulthood. Furthermore, most aggravation from deformity is progressive in the grow spurt period. It is well known that even spinal orthosis for scoliosis is limited to prevent the exacerbation $[14,15]$. Thus, the physiatrist should consider the aspect of comfort, rather than aggressive correction of deformities. In that sense, the custom molded fitting chair would be a more suitable instrument for severely disabled children for its comfort. Although it is not significant statistically, a slight exacerbation of the deformity might be significant clinically.

The limitation of study is as follows. Without the chair, the deformities might become worse compared to the state with the chair. To confirm this problem, comparing between patients with the chair and without the chair in a controlled study is needed. However, an ethical problem arises in that the patients without the chair may endure discomfort.

The growth spurt is one of the most critical variables that affect numerous musculoskeletal deformities. Pro- gression of scoliosis is greatest during the growth spurt and may continue after skeletal maturity, especially when associated with pelvic obliquity [12]. Therefore, it is important to separate the children with growth spurts. Although this was done, the number of subjects after the growth spurt is too little to acquire significant results. Further investigation should be done with more subjects who experience the growth spurt period.

In our study, it was found that the custom molded fitting chair had the effect of preventing the progression of coxa valga in severely disabled children. In addition, there was no significant aggravation of the Cobb's angle or Reimers migration percentage. However, a considerable number of patients did not show aggravation of deformities. Thus, we can expect the use of the custom molded fitting chair to delay rapid aggravation of musculoskeletal deformity.

\section{CONFLICT OF INTEREST}

No potential conflict of interest relevant to this article was reported.

\section{ACKNOWLEDGMENTS}

This study was supported by a grant from Inha University.

\section{REFERENCES}

1. Morrell DS, Pearson JM, Sauser DD. Progressive bone and joint abnormalities of the spine and lower extremities in cerebral palsy. Radiographics 2002;22:25768.

2. Thometz JG, Simon SR. Progression of scoliosis after skeletal maturity in institutionalized adults who have cerebral palsy. J Bone Joint Surg Am 1988;70:1290-6.

3. Madigan RR, Wallace SL. Scoliosis in the institutionalized cerebral palsy population. Spine (Phila Pa 1976) 1981;6:583-90.

4. Miller F, Bagg MR. Age and migration percentage as risk factors for progression in spastic hip disease. Dev Med Child Neurol 1995;37:449-55.

5. Saito N, Ebara S, Ohotsuka K, Kumeta H, Takaoka K. Natural history of scoliosis in spastic cerebral palsy. Lancet 1998;351:1687-92. 
6. Gajdosik CG, Cicirello N. Secondary conditions of the musculoskeletal system in adolescents and adults with cerebral palsy. Phys Occup Ther Pediatr 2001;21:49-68.

7. Greiner KA. Adolescent idiopathic scoliosis: radiologic decision-making. Am Fam Physician 2002;65:181722.

8. Reimers J. The stability of the hip in children: a radiological study of the results of muscle surgery in cerebral palsy. Acta Orthop Scand Suppl 1980;184:1-100.

9. Noble PC, Alexander JW, Lindahl LJ, Yew DT, Granberry WM, Tullos HS. The anatomic basis of femoral component design. Clin Orthop Relat Res 1988;(235): 148-65.

10. Suh YJ, Kim ES, Park ES, Park HS, Yoon YK, Cho SR. Attenuation of spinal curvature and pelvic obliquity by body shape molded inner system in cerebral palsy with non-fixed scoliosis. J Korean Acad Rehabil Med
2011;35:259-64.

11. Hoffer MM. Management of the hip in cerebral palsy. J Bone Joint Surg Am 1986;68:629-31.

12. Ferguson RL, Allen BL Jr. Considerations in the treatment of cerebral palsy patients with spinal deformities. Orthop Clin North Am 1988;19:419-25.

13. Foroohar A, McCarthy JJ, Yucha D, Clarke S, Brey J. Head-shaft angle measurement in children with cerebral palsy. J Pediatr Orthop 2009;29:248-50.

14. Chase AP, Bader DL, Houghton GR. The biomechanical effectiveness of the Boston brace in the management of adolescent idiopathic scoliosis. Spine (Phila Pa 1976) 1989;14:636-42.

15. Aubin CE, Dansereau J, de Guise JA, Labelle H. Rib cage-spine coupling patterns involved in brace treatment of adolescent idiopathic scoliosis. Spine (Phila Pa 1976) 1997;22:629-35. 